\title{
Proyecto Hiperión: Programa de conocimiento y gestión de los procesos mentales a través del cuerpo ${ }^{1}$
}

Hiperion Project: a program of understanding and management of mental processes through the body.

Jesús Zafra Márquez

IES Sto. Domingo

El Puerto de Santa María (Cádiz)

jesus.zafra@iessantodomingo.com

\section{Resumen}

En el presente artículo damos a conocer el Proyecto Hiperión, desde sus elementos básicos hasta su implementación en estudiantes de enseñanza secundaria y bachillerato de la provincia de Cádiz. Este proyecto de innovación docente nace a partir del estudio de contenidos procedentes de la neurociencia y el yoga, con el objetivo de iniciar a los jóvenes en el conocimiento y gestión de algunos de los procesos mentales básicos. Creemos que la mejor forma de favorecer el crecimiento y la evolución personales es a través del desarrollo de la sensibilidad, la atención y la capacidad de observación de nuestra actividad mental. Hacemos también referencia a los principios teóricos que sustentan esta propuesta innovadora y las perspectivas de futuro que se presentan a partir del trabajo realizado hasta el momento.

Palabras clave: Proyecto Hiperión, sensibilidad, atención, neurociencia, educación, cuerpo.

\section{Abstract}

In this article we present the theoretical foundation and implementation of the Hyperion Project among students in the province of Cádiz attending mandatory and pre-university high school. This teaching innovation project was developed combining principles of

${ }^{1}$ Recibido: 28/02/2020 Evaluado: 16/02/2020 Aceptado: 13/04/2020 
neuroscience and yoga, with the aim of introducing young people to the knowledge and management of some of the basic mental processes. Our premise is that the best way to foster personal growth and development is by building awareness of our senses, improving our attention span, and observing our mental activity. Reference is also made to the theoretical principles sustaining this proposal and to the future prospects from the work carried out so far.

Key words: Hiperion Project, sensibility, attention, neuroscience, education, body.

\section{Introducción}

En el presente artículo describiremos en detalle los antecedentes, el marco epistemólogico, objetivos y contenidos del Proyecto Hiperión, programa para el conocimiento y Gestión de los procesos mentales básicos a través del cuerpo en educación secundaria y bachillerato.

Este proyecto empieza como una inquietud personal, resultado del interés de un profesor de filosofía de Educación Secundaria por la filosofía de la mente, las neurociencias y el estudio del yoga. Esta inclinación inicial por conocer el funcionamiento del sistema nervioso y la mente humana ha ido progresivamente creciendo, para convertirse en un proyecto colectivo que está empezando a aplicarse en algunos centros públicos de la provincia de Cádiz. Las primeras prácticas surgieron, hace ya diez años, casi como un juego, con el diseño de breves ejercicios en los que experimentábamos con nuestro cuerpo escuchando el ritmo del corazón, jugando con la percepción o disfrutando del silencio en grupo. Estos ejercicios se incorporaban al final de nuestras clases de filosofía. La cálida acogida que tuvieron estos tímidos intentos de incorporar las primeras prácticas, sirvieron como acicate para continuar estudiando y diseñando ejercicios que eran inmediatamente puestos a prueba en el seno de clase.

Así es como los primeros ejercicios fueron creciendo en número y dando lugar a diferentes versiones de un proyecto que con el tiempo ha ido cambiando de estructura e incorporando nuevos apartados.

En el año 2017 entendemos que ya disponemos de una propuesta digna de ser difundida y compartida con otros compañeros. El trabajo realizado hasta el momento recibe el nombre de Proyecto Hiperión: programa de conocimiento y gestión de los procesos mentales a través del cuerpo, en honor al personaje literario creado por el poeta romántico alemán Hölderlin en su novela epistolar homónima (1795). Comienza entonces una fase de difusión en la que invitamos a otros compañeros a conocer y aplicar algunos de los contenidos de este proyecto en sus aulas.

En 2017, gracias a la colaboración del Centro del Profesorado de Cádiz, impartimos el primer curso en torno al Proyecto Hiperión. La respuesta por parte del profesorado fue muy favorable. Con el primer curso tuvimos la ocasión de constatar que gran parte de las actividades que habían sido concebidas para ser aplicadas en las aulas del IES Santo Domingo de El Puerto de Santa María (Cádiz), en concreto en las clases de filosofía del 
profesor responsable de su diseño, también eran aplicables por otros compañeros y compañeras con alumnado de educación primaria o educación especial. Compartir nuestra experiencia resultó enormemente satisfactorio y muy motivador. Comprobamos que los resultados observados en el aula en la fase de diseño del proyecto, eran replicables en otras aulas y otros centros.

Producto de este impulso, el proyecto vuelve a crecer con la incorporación de dos nuevos bloques de contenido en 2018, que se suman al bloque inicial compuesto por actividades para trabajar las facultades mentales básicas (“pensamiento lateral” y "respiración consciente”). Nos planteamos entonces profundizar en nuestra tarea con la creación de grupos de trabajo que favorezcan la formación del profesorado y la aplicación integral del proyecto en otros centros educativos de la provincia.

Llegados a este punto, en el presente curso (2019-20) comienza una nueva fase en la evolución del Proyecto Hiperión, al iniciar la colaboración con las Universidades de Sevilla y Cádiz, que nos permitirá observar con el mayor rigor a nuestro alcance los resultados de la implementación de esta propuesta en las aulas, en concreto en el primer ciclo de enseñanza secundaria.

Nos anima el hecho de vernos envueltos en un proceso que cambia y se transforma de forma constante. La reciente incorporación de compañeros y de centros nos hace pensar que nos encontramos al comienzo de una prometedora andadura en la que cada paso abre nuevos caminos, presenta nuevos retos que se convertirán en la guía que habrá de orientar esfuerzos venideros.

\section{Estado de la cuestión}

Como todo mecanismo, el paso del tiempo y los cambios que tienen lugar en el entorno, hacen necesaria la aplicación de continuos ajustes en el seno del sistema educativo, con el fin de optimizar su funcionamiento. Estas intervenciones se plantean desde frentes muy diversos.

A la hora de incorporar estrategias innovadoras en el aula, se puede seguir un modelo topdown, es decir, a través de aportaciones venidas desde las instituciones educativas, o bien bottom-up (Harland Mills y Niklaus Wirth, 1970), partiendo de propuestas diseñadas e implementadas a partir de la experiencia en las aulas. Nuestra propuesta se encuadra en el segundo grupo, pero no es la única. Son bastantes los intentos por poner al servicio de la educación aportaciones venidas de campos tan diversos como la neurociencia, la música, el deporte, el teatro..., con el objetivo de hacer más productivo, eficiente y práctico el aprendizaje en la escuela. Nuestra propuesta es una más, con sus peculiaridades, a las que nos referiremos más adelante, pero solo una entre bastantes otras.

Presentaremos tres experiencias que tienen cierta similitud con nuestro Proyecto y que se están llevando a cabo en distintos puntos de nuestro país. En algunos colegios de Zaragoza se está aplicando el programa Aulas Felices (catedu.es/psicologíapositiva), con resultados muy interesantes. Desde “Aulas Felices” diseñan su intervención a partir de dos pilares fundamentales: la atención plena y la educación de las fortalezas personales. El grupo SATI 
parte del enfoque propuesto por la llamada Psicología Positiva (Linley Stephen, Harrington y Wood, 2007) al plantear una idea que encontramos en los orígenes de la filosofía griega, la felicidad como principal objetivo de la existencia humana. Los ideólogos de esta escuela de psicología plantean, como ya hicieran mucho tiempo antes autores como Sócrates, Platón, Aristóteles, Epicuro, Zenón, Séneca..., que esta meta necesita de un aprendizaje y que es la educación el medio idóneo por el que han de transmitirse las herramientas para abordar una búsqueda de tal calibre.

En Cataluña encontramos el programa TREVA (Técnicas de Relajación Vivencial Aplicadas al Aula). En este caso, el profesor Luis López González ha diseñado un plan de intervención en las aulas basado en diferentes modelos de relajación adaptados al ámbito educativo (López, González, 2016). Este proyecto se está aplicando a alumnado desde los 3 a los 18 años.

Terminamos refiriéndonos a otra experiencia especialmente interesante, se trata de la investigación promovida desde la Universidad de Almería en la que se ha querido comprobar el efecto que tiene la formación en técnicas de meditación sobre profesorado de educación especial. Esta última experiencia nos parece especialmente interesante por poner de manifiesto el resultado que este tipo de intervenciones tiene sobre los educadores (Franco, Mañas, Justo, 2009).

La nuestra es solo una más entre las diferentes propuestas que están siendo aplicadas en estos momentos y que ponen de manifiesto que existe una sensibilidad en el contexto educativo que está empezando a orientar la praxis educativa en esta dirección. No es casualidad que todas estas nuevas formas de trabajo estén coincidiendo en el tiempo, también la ciencia, en particular la medicina y la neurociencia, empiezan a dirigir su foco de atención hacia cuestiones que vienen siendo objeto de estudio en oriente desde hace siglos. En este sentido podemos decir que la transformación que se está produciendo en la educación es un reflejo de lo que está ocurriendo en las ciencias de la mente y de la salud.

\section{Marco Teórico}

No resulta sencillo determinar cuál ha de ser el campo de acción propio de la enseñanza reglada. Parece poco cuestionable, por ejemplo, que las enseñanzas adquiridas en la escuela tienen muy poca repercusión a la hora de compensar las desigualdades sociales (Calero, 2007). Tampoco parece que las escuelas ofrezcan soluciones en la gestión de posibles desequilibrios emocionales. Trastornos como el estrés, la ansiedad y la inestabilidad psicológica en general, lejos de reducirse crecen de manera alarmante en la sociedad tanto como en el seno de los centros educativos (Barraza, 2005; Maturana, 2015), sin que el sistema se plantee siquiera el tratamiento de esta problemática como una cuestión propia.

En general, pensamos, existe la creencia de que estas cuestiones están fuera de los objetivos de la educación. Existe casi un consenso al pensar que realidades como la lucha contra la desigualdad social pertenecen exclusivamente al ámbito de la economía y la política, mientras que los desequilibrios emocionales y mentales al de la salud. Resulta extraño plantear que la educación tenga algo que decir a este respecto. 
Creemos que no es así. La vida es el marco en el que se circunscriben el resto de las realidades humanas (Ortega y Gasset, 1923; Nietzsche, 1997). Todo lo que nos acontece tiene lugar porque estamos vivos: aprender a vivir debe ser el principal de los objetivos de nuestras escuelas, aquel al que se supediten todos los demás. Y la vida, al igual que todo posible acontecer, tiene lugar a través del cuerpo (Merleau-Ponty, 1985). Nuestros cuerpos se convierten en la realidad esencial, el medio en el que todo ocurre. Más aún, el medio que condiciona todo lo que ocurre. Aprender a vivir supone, entre otras cosas ocuparnos de nuestro cuerpo. Sin embargo, el cuerpo como generador de experiencia y condicionante de la actividad mental, rara vez se convierte en objeto de tratamiento en las aulas.

Desde los comienzos de nuestro trabajo con el Proyecto Hiperión tuvimos claro que el conocimiento y gestión de nuestro cuerpo se convertiría en el vehículo a través del cual introducirnos en el estudio de nosotros mismos y de la relación con nuestro entorno. Esta aparentemente inocente tarea ofrece un vastísimo potencial. Al iniciarnos en la observación, conocimiento e intervención en los procesos mentales básicos, nos embarcamos en una tarea profundamente revolucionaria. Y esto es así, porque modificar, optimizar el uso que hacemos de nuestro sistema nervioso, de nuestra atención, sensibilidad o respuestas emocionales, nos ofrece la posibilidad de intervenir en la imagen que construimos acerca de nosotros mismos y la realidad en la que nos ha tocado vivir.

El tipo de ejercicios que forma parte de este proyecto ha sido diseñado tomando como inspiración algunas de las investigaciones más relevantes en el ámbito de la neurociencia (Ma,Yue et al. 2017; Conde, 1999; Cea et al., 2005: Cea, González-Pinto, Cabo González 2010, Broderick, 2013). Por otro lado, tan inspiradora como la neurociencia, ha sido el estudio de las propuestas de la tradición yóguica (Henson, 2016; Ruiz Lázaro, 2016; Davidson, 2012). En ambas tradiciones (occidental-neurociencia y oriental-yoga), las prácticas propuestas comparten el énfasis puesto en el entrenamiento de las diferentes modalidades de atención como vehículo que hace posible modificar la naturaleza de la experiencia cotidiana y, consiguientemente, la actividad del sistema nervioso que la sustenta.

En torno a la problemática del cuerpo humano como sede de todos los procesos físicos y mentales a la base de la experiencia humana, encontramos un campo en el que confluyen tanto la tradición yóguica, como la filosofía y la ciencia occidentales. Ambas tradiciones son el fundamento en el que nos hemos apoyado para diseñar el conjunto de prácticas que configuran el Proyecto Hiperión.

En el apartado relacionado con el conocimiento y gestión de las emociones hemos tomado como referencia los estudios del neurocientífico Davidson (2012). La trayectoria de este autor encaja perfectamente con nuestros intereses por varias razones. Por un lado, su experiencia en el estudio del efecto de las prácticas meditativas en el sistema nervioso, por otro, el hecho de que su trayectoria profesional haya estado vinculada con la investigación de la actividad emocional durante los últimos 20 años. También nos ha interesado especialmente la noción de perfil emocional del cerebro (Davidson, 2012). En el apartado de las emociones han resultado también muy valiosas las aportaciones de James (1994), su concepción de la emoción como resultado de la respuesta corporal ante la realidad circundante. Destacamos 
también las aportaciones de Ekman al ocuparnos de la interpretación de las emociones a través del rostro (Ekman 2011, 2014). En el bloque dedicado al pensamiento lateral nos han servido como referentes Bono $(1989,1992)$ y Gardner (1985). Con esto nombramos solo a algunos de los autores en los que nos hemos inspirado al diseñar las prácticas contenidas en el proyecto. No obstante, la lista de científicos que han servido de sustento teórico en el proceso de documentación y creación del Proyecto Hiperión sería bastante mayor (Ramachadran, 2012; Sacks, 2006; Goldberg, 2002; Damasio, 2006; Candace Pert, 1999...)

En lo que respecta a las aportaciones procedentes de la tradición oriental, la mayor parte de los ejercicios de respiración consciente contenidos en el proyecto, tienen su origen en la ancestral práctica del pranayama, tal y como ha sido transmitida desde la Bihar School of Yoga. (https://cutt.ly/kt1ReTb.). La mayoría de los tratados sobre yoga que nos han servido de apoyo han sido publicaciones editadas por este relevante centro de estudios, ubicado en el norte de La India, entre los que destacamos algunas de las obras de su fundador, S. Satyananda Saraswati.

Puede resultar extraño en un trabajo de estas características no hacer una mención especial al Mindfulness. Entendemos el mindfulness como una lúcida y correcta síntesis de una parte de las enseñanzas contenidas en la tradición yóguica de origen tibetano e hindú. Su principal mérito reside en haber servido de vehículo para la difusión de estos conocimientos y también haber ofrecido la posibilidad de someterlos al riguroso filtro que supone la revisión de algunas de sus propuestas por parte de la ciencia occidental. Pero, a pesar de las numerosísimas publicaciones realizadas en los últimos veinte años, consideramos que son muy pocas las novedades aportadas con respecto a la tradición en la que se inspira. Esta es la razón por la que, tras haber consultado algunos manuales procedentes de esta corriente de psicología, desde el principio de nuestro trabajo nos inclinamos por dirigir nuestra mirada a la fuente original.

\section{Descripción del Proyecto Hiperión: programa de conocimiento y gestión de los procesos mentales a través del cuerpo}

\section{Objetivos del proyecto Hiperión.}

Nos referiremos a continuación a algunos de los objetivos que perseguimos con este proyecto:

a. Proporcionar herramientas que favorezcan una interpretación constructiva de nosotros mismos y de la realidad que nos rodea. Iniciar al alumnado en el conocimiento de los procesos que conforman nuestra actividad mental desde una perspectiva vivencial más que teórica, puede ser el camino para educar a individuos más sanos y felices.

b. Ofrecer a los alumnos y alumnas un conocimiento que les ayude a ser más conscientes y gestionar de forma más saludable su compleja vida interior.

c. Ayudar a optimizar los recursos mentales invertidos por los componentes de la clase (alumnado y profesorado), al favorecer que trabajemos con una atención más centrada y sostenida. 
d. Trabajar con la atención, la concentración, la memoria o la percepción, experimentar sus limitaciones y la capacidad de optimizar su funcionamiento, necesariamente ha de repercutir en la capacidad para aprender, en aquellos alumnos y alumnas a los que seamos capaces de transmitir estos contenidos.

e. Profundizar en el conocimiento y gestión de la actividad emocional propia a través del entrenamiento y mejora de la sensibilidad (percepción, pensamiento, emociones). (Davis, 1980).

f. Experimentar la estrecha relación existente entre la actividad emocional y el sistema respiratorio. De este modo esperamos aprender a afrontar la impulsividad, fuente de gran cantidad de conflictos en todas las etapas de la vida, pero especialmente en la adolescencia.

g. Ofrecer al profesorado que participa en la implementación del proyecto en las aulas, instrumentos que les permitan mejorar el clima de clase y el proceso de enseñanzaaprendizaje.

\section{Metodología}

Las prácticas que forman parte de nuestro proyecto se enmarcan dentro de la categoría de conocimientos "to know how" (Foray y Lundvall, 1996), es decir, se trata de un tipo de aprendizaje que no se puede transmitir de forma teórica (no podemos aprender a atarnos los zapatos de otra forma que practicando, actuando). Por esta razón la metodología de nuestra propuesta es fundamentalmente práctica. Esto no significa que no haya una transmisión de contenido teórico. Pero cuando este aparece, siempre está al servicio de la experiencia a la que acompaña. El lugar de la teoría es principalmente ayudarnos a enfocar la experiencia. Conocer el fundamento sirve de guía para alumbrarnos en la práctica y favorecer así que tenga lugar el acto de conocimiento.

El único vehículo, la herramienta de aprendizaje, es nuestro cuerpo. Se trata de aprender a gestionar nuestro cuerpo utilizando el cuerpo.

Nuestra propuesta pretende ser variada, amena, coherente y versátil:

Variada, porque pensamos que la clave para obtener la complicidad de los participantes en las actividades propuestas es procurar evitar el tedio. El aburrimiento es el peor enemigo del tipo de aprendizaje que estamos proponiendo. Por esta razón, a pesar de que nos centraremos en trabajar un número reducido de capacidades mentales, cada uno de los bloques integra prácticas diferentes, pero que ejercitan procesos mentales semejantes.

Coherente, porque hemos puesto especial interés en el modo de estructurar los contenidos.

Versátil. El hecho de que todas las prácticas ejerciten más de una de las capacidades mentales con las que trabaja este proyecto, nos permite encajar (o al menos intentarlo), en contextos muy diferentes. 


\section{Estructura de las sesiones}

Las sesiones de trabajo tienen una duración de entre diez y quince minutos y generalmente se dividen en tres fases (la duración de cada parte depende de la actividad y las características del grupo):

- Fase inicial. Presentación de los contenidos de la ficha teórica que acompaña a la actividad. Se suele presentar al principio de la misma y hemos de intentar que sea precisa, escueta y clara.

- Desarrollo. La mayoría de las actividades propuestas se desarrollan en completo silencio. Esta fase ocupa la mayor parte del tiempo destinado a la actividad. Cuando conseguimos transmitir a los alumnos la motivación suficiente para "entrar en el juego”, se crea en el aula una atmósfera de silencio y concentración que nos arrastra a todos.

- Cierre. Reservamos (casi siempre) los últimos minutos de la sesión para poner en común lo experimentado a lo largo de la práctica (generalmente la experiencia propuesta nos permite poner a prueba, reforzar, utilizando nuestro cuerpo como laboratorio, los contenidos teóricos presentados al inicio de la sesión).

\section{Contenidos}

Hemos distribuido los contenidos del Proyecto Hiperión en tres bloques:

A. Entrenamiento de las facultades mentales básicas. A la hora de realizar la selección procuramos que la lista no fuera demasiado larga, pensando que un campo de acción reducido nos permitiría trabajar con más profundidad. El estudio de las fuentes a las que nos hemos referido en la fundamentación teórica, nos llevó a inclinarnos por las siguientes facultades como los procesos básicos sobre los que se sustenta la actividad mental humana:

1.1.Sensación y pensamiento. 10 sesiones.

1.2.Memoria. 5 sesiones.

1.3.Atención. 10 sesiones.

1.4.Emoción. 11 sesiones

1.5.Interpretación de rostros. 5 sesiones.

1.6.Visualización. 11 sesiones.

B. Respiración consciente. Multitud de estudios científicos evidencian la relevancia de la respiración consciente como herramienta privilegiada para gestionar procesos mentales como la atención, observación e intervención en la actividad emocional o control del estrés (Ravinder, 2015). Podemos señalar dos razones fundamentales que justifican suficientemente la incorporación de ejercicios de respiración consciente en este proyecto: 
- En todos los ejercicios hemos de cuantificar la duración de cada fase de la respiración. Al introducir una ratio en la duración de las partes de cada ciclo respiratorio (inhalación, retención y exhalación) y al sugerir a los participantes que mientras realizan los diferentes ejercicios propuestos cuenten el número de rondas realizadas, además de trabajar con la capacidad respiratoria, también estamos ejercitando la atención.

En un principio, la mayoría de los participantes tiene serias dificultades para realizar las dos tareas a la vez. Cuando esto ocurre, sugerimos que utilicen los dedos de la mano para contar las rondas. Pasado un tiempo, todos los participantes comentan que consiguen realizar los ejercicios con mayor facilidad, sin necesidad de apoyarse en los dedos de las manos para contar.

- El segundo motivo reside en que la respiración está estrechamente relacionada con la activación del nervio vago (Cea, 2002, 2005).

Entre otras funciones del nervio vago, está la de activar el sistema parasimpático a través de la liberación de acetilcolina, que conduce al organismo a un estado de relajación, por lo que la posibilidad de intervenir en su funcionamiento nos proporciona, entre otras cosas, una extraordinaria herramienta contra el estrés.

Los ejercicios de respiración contenidos en este apartado del proyecto son los siguientes:

- Respiración abdominal.

- Respiración intercostal.

- Respiración yóguica.

- Kapalabhati.

- Nadhi Shodana.

\section{Pensamiento lateral}

En nuestras escuelas se favorece una forma de razonar propia del pensamiento científico que autores como Horkheimer y Adorno (2007) han llamado pensamiento instrumental. Se trata de una forma de procesar la información que tiende a seguir un patrón casi fijo: parte de los objetivos a conseguir y posteriormente diseña maneras de alcanzar estos objetivos. Esta forma de razonar ha sido muy productiva a lo largo de la historia. El conocimiento científico aspira a un saber universal, para lo cual es preciso formular leyes capaces de subsumir bajo el mismo paraguas la enorme diversidad de lo existente. El problema está en que el pensamiento instrumental, que de tantísima utilidad ha sido para el conocimiento científico, se ha acabado erigiendo en la única forma de pensamiento fiable y válido.

Tomar decisiones requiere de creatividad, intuición, sentido lúdico y, a veces, incluso cierta dosis de temeridad. Este es el terreno en el que se mueve el pensamiento lateral. Con el tipo de actividades que proponemos en este apartado del proyecto, invitamos a pensar sin miedo al ridículo, a forzar las líneas de lo que habitualmente consideramos posible o imposible. Porque en muchas ocasiones es la realidad la que supera estos límites. El pensamiento lateral 
está abierto a la sorpresa, a la novedad, al cambio, características estas definitorias de la realidad que nos rodea. Esto es lo que lo hace tan útil en determinados contextos.

No se trata de demonizar una forma de pensamiento para exaltar a la otra. Buscamos más bien transmitir que la realidad es mucho más compleja y divertida de lo que nos muestran los manuales académicos y que para vivir necesitamos ser flexibles y entender qué modalidad de pensamiento es la adecuada a cada contexto. Familiarizarnos con el pensamiento lateral puede ser una vacuna contra el dogmatismo y la estrechez mental.

Hemos clasificado las actividades de este apartado sobre pensamiento lateral en tres categorías:

- Acertijos: ejercicios muy diversos que proponen resolver problemas utilizando vías poco usuales o evidentes.

- Ejercicios con imágenes. En este caso trabajamos con fotografías, solicitando interpretaciones acerca de lo que representan, imaginando los pensamientos de alguno de los protagonistas, proponiendo desenlaces posibles...

- Ejercicios con palabras. Ofrecemos palabras elegidas aleatoriamente y proponemos diferentes dinámicas que nos hagan imaginar criterios para asociarlas.

Referirnos con detalle al tipo de prácticas contenidas en el proyecto nos llevaría a rebasar las dimensiones de este artículo. En la siguiente dirección Web aparecen ejemplos de algunas de las prácticas incluidas en los tres apartados del proyecto a los que nos acabamos de referir, explicadas con detalle: https://cutt.ly/vt1RaeT

Como comentábamos en el apartado anterior, consideramos que el dinamismo es una virtud más que una limitación.

No hay una forma exclusiva de distribuir las prácticas. Más bien al contrario, cada centro, cada profesor o profesora, debe modificar esta propuesta dependiendo de las características del alumnado y del número de personas implicadas en su implementación.

\section{Implementación del proyecto}

Las modalidades de aplicación son tan variadas como centros dispuestos a implementar el proyecto. Los niveles educativos en los que se aplique esta forma de trabajo dependen de la demanda de cada centro, así como de los recursos humanos disponibles. En el caso de nuestro centro, desde hace dos cursos nos estamos centrando en todos los grupos de $1^{\circ}$ y $2^{\circ}$ de ESO, cuatro sesiones semanales: dos sesiones de entrenamiento de las facultades mentales básicas, una de pensamiento lateral y otra de respiración consciente.

Dedicamos el primer trimestre del curso a la formación del profesorado y el grupo de alumnos y alumnas que se van a encargar de dirigir las sesiones de respiración en el aula. Las sesiones de respiración consciente (una o dos sesiones semanales, dependiendo de la elección del alumnado que forma el grupo) son dirigidas por los propios alumnos y alumnas sin la 
participación de los profesores. Una vez realizada la formación, estamos en condiciones de empezar el trabajo en las clases durante el resto del curso (dos trimestres).

No es necesario reservar un tiempo específico para la práctica en el horario: la duración de las actividades es de unos quince minutos, de manera que basta con dedicar un espacio al principio o al final de la clase en la que se va a implementar la actividad, con independencia de la materia que se esté impartiendo. Esto hace que la aplicación resulte fácil para los centros interesados, al no ser necesario hacer encajes en los horarios de los grupos.

Esta forma de proceder no sería posible sin la complicidad de todos los miembros del claustro, que, participen o no en la implementación del proyecto, han de estar convenientemente informados. En nuestro instituto, este proyecto está integrado en el Plan de Centro. La participación del profesorado es voluntaria, pero también la del alumnado. No obstante, es absolutamente excepcional que algún alumno o alumna manifieste su intención de no participar en las actividades propuestas.

Las sesiones de entrenamiento de las facultades mentales básicas y pensamiento lateral son dirigidas por profesorado que se ha formado durante el primer trimestre del curso y que continúa formándose a través de la participación en un grupo de trabajo que se reúne mensualmente. Sin embargo, en el caso de las prácticas de respiración consciente, en cada grupo de clase hay un pequeño equipo de cuatro o cinco alumnos y alumnas que han dedicado durante el primer trimestre un recreo semanal a recibir la formación adecuada para que sean ellos los que se encarguen de enseñar a sus compañeros de clase las diferentes técnicas de respiración. Esta forma de trabajar está siendo muy satisfactoria para todos. Tanto el alumnado que dirige las sesiones como el que las recibe hacen posible que el proceso de enseñanza-aprendizaje tenga lugar de forma fluida y amena.

\section{Perspectivas de futuro}

Tenemos la sensación de encontrarnos al principio del viaje. A pesar de haber empezado a estudiar, diseñar y aplicar las primeras versiones del proyecto hace ya unos años, es ahora cuando estamos fomentando, gracias a la colaboración del CEP de Cádiz, la creación de redes formadas por profesores y profesoras interesados en aplicar esta forma de trabajo en sus aulas.

Más interesante aún es el hecho de que, más allá de profesoras y profesores a nivel particular, sean los equipos educativos y departamentos de orientación, el centro educativo en bloque, los que asumen la inclusión de estos contenidos en el seno de su plan de centro. Ésta es la etapa del camino que estamos iniciando en estos momentos. Oportunidades como la que se nos presenta al contar con la posibilidad de compartir nuestra experiencia en esta revista, o la reciente creación de grupos de trabajo, con profesorado de varios centros de la provincia dispuestos a incorporar las prácticas contenidas en el proyecto Hiperión en sus clases, nos hacen encarar el futuro inmediato con ilusión. 
Es cuestión de tiempo que las administraciones educativas responsables de sustentar nuestro trabajo entiendan la relevancia de la transformación que está empezando a llevarse a cabo necesita de su colaboración.

Nuestra experiencia es que por parte de la administración educativa se están ofreciendo cauces para la formación del profesorado, pero falta la flexibilidad necesaria para apoyar la implementación de propuestas innovadoras. Un ejemplo de esta flexibilidad hacia los centros, sería la dotación de horas extra en la asignación de la carga horaria, que permita una dedicación a este tipo de iniciáticas por parte del profesorado. Con esto se evitaría la sobrecarga de trabajo que supone para el profesorado implicado la participación en este tipo de iniciativas. Hasta el momento todos estos esfuerzos se están realizando contando con las horas de trabajo que aportan de manera desinteresada los profesores y profesoras al margen de su horario lectivo.

Somos los profesionales que trabajamos dentro de las aulas los que experimentamos la necesidad de emprender cambios para abordar algunos de los desequilibrios existentes en nuestros centros: el estrés, problemas de ansiedad y atención entre nuestro alumnado no han hecho más que crecer en los últimos años de forma preocupante, sin que hayamos sido capaces de diseñar una respuesta a esta realidad.

Por otro lado, continúa siendo una asignatura pendiente la acogida en el seno del sistema de la legión de niños y niñas procedentes de los sectores más desfavorecidos de nuestras ciudades. La fisura existente en la sociedad, lejos de ser paliada desde el sistema educativo, se reproduce. Los hijos e hijas de las personas que viven al margen del sistema, continúan la margen dentro de nuestras aulas, acumulando fracasos, generando y sufriendo una conflictividad a la que rara vez sabemos hacer frente.

La satisfacción que resulta del recorrido realizado hasta el momento y la ilusión ante los retos presentes nos dan fuerza para plantearnos hacer frente a estas problemáticas.

Somos conscientes de que, tras el trabajo realizado hasta el momento, urge promover una investigación seria y rigurosa que tenga como objeto observar las repercusiones que las intervenciones propuestas por el Proyecto Hiperión tienen en el alumnado y los centros en los que se aplica. Esta es la fase en la que nos encontramos en estos momentos. Un esfuerzo de este calibre necesita emprenderse desde el máximo rigor posible.

Con este afán hemos buscado la colaboración de las universidades de Sevilla y Cádiz, como ya comentamos en la introducción. El Proyecto Hiperión se encuadra en el seno de una tesis doctoral (inscrito en el programa "Psicología” y la línea de investigación “Aprendizaje, Neurociencia y Evolución” de la Universidad de Sevilla), que pretende observar los efectos de las prácticas que lo componen sobre el alumnado y el proceso de enseñanza-aprendizaje en el grupo de clase (clima de clase, relación entre el alumnado, resultados académicos, disruptividad en el aula...). Este estudio se está llevando a cabo a través de la aplicación de las herramientas y procedimientos propios de la metodología científica.

Por otro lado, como un apartado más de la investigación iniciada al abrigo de la mencionada tesis doctoral, estamos trabajando con dos grupos de alumnos y alumnas pertenecientes a los 
IES Santo Domingo y Las Banderas de El Puerto de Santa María (Cádiz), que presentan diferentes tipos de problemáticas relacionadas con el déficit de atención (TDAH, atención dispersa, falta de control de impulsos...). Hemos diseñado una intervención a partir de la adaptación de prácticas contenidas en el Proyecto Hiperión (técnicas de respiración, prácticas para ejercitar la atención, visualización y actividad emocional, interpretación del lenguaje no verbal) y el manejo de una App diseñada “ex profeso”, para intentar promover cambios encaminados a mejorar las capacidades atencionales de los adolescentes participantes.

Como consecuencia de los cursos de formación para profesores, se han formado varios grupos de trabajo compuestos por profesoras y profesores de centros de distintas localidades de la provincia (Conil, Chiclana, Rota y El Puerto de Santa María), con el objetivo de continuar con la formación, ofrecer apoyo a sus componentes y plantear un una línea de acción común a través de una reunión mensual. Una vez consolidada está red de profesores y profesoras, en un futuro próximo se nos presenta el reto de abordar el objetivo de reducir la conflictividad en nuestros centros.

El principio que mueve todas las propuestas en las que nos hemos embarcado, es que el conocimiento de nuestros cuerpos y el entrenamiento de los procesos mentales que están a la base de nuestra actividad vital (fundamentalmente cognición y emociones), nos ofrecen la posibilidad de favorecer la evolución y el crecimiento personales.

Pensamos que tenemos entre manos una tarea titánica, pero con un potencial trasformador enorme, tanto a nivel individual como en los grupos en los que seamos capaces de incorporar el trabajo con este tipo de conocimiento (hasta el momento presentes solamente de manera residual en los centros de enseñanza).

Atrevernos a pensar lo imposible es un estadio ineludible para transformar la realidad.

\section{Conclusiones}

Al diseñar esta propuesta de intervención en las aulas, nos mueve la ilusión de pensar que estamos proponiendo al sistema educativo la posibilidad de ofrecer a quienes en él se forman, un conocimiento que les va a ayudar a ser más conscientes y gestionar de forma más saludable su compleja vida interior.

Iniciarse en el conocimiento de los procesos que conforman nuestra actividad mental desde una perspectiva vivencial más que teórica, puede ser el camino para educar a individuos más sanos y felices.

El tipo de trabajo que proponemos nos enseña que somos nosotros los que, en última instancia, procesamos todas las influencias llegadas de nuestro entorno para integrarlas de una forma u otra en nuestro organismo. No caer en la cuenta de este hecho tan relevante y a menudo olvidado, nos convierte en marionetas que se mueven a merced de lo que ocurre en el exterior. Dedicar varias sesiones semanales a ejercitarnos en observar e intervenir en todos esos procesos de los que resulta nuestra actividad mental, puede ser el camino para educar a individuos más libres. 
El estrés y la ansiedad son problemas habituales entre el profesorado debido a la naturaleza de nuestro trabajo (Martínez-Otero, 2003). Las técnicas con las que tendremos que familiarizarnos al incorporar en nuestras aulas los contenidos que proponemos con este proyecto, pretenden ofrecer herramientas de conocimiento y control de nuestra actividad mental y otros procesos fisiológicos asociados que, de ser asimilados, han de beneficiar al conjunto de la comunidad educativa.

\section{Referencias}

Barraza, A. (2005). Característica del estrés académico de los estudiantes de educación media superior. INED, (4), 15-20. Universidad Pedagógica de Durango.

Bayón, M. y Martínez, J. (2008). Plasticidad cerebral inducida por algunas terapias aplicadas en el paciente con ictus. Rehabilitación, Volume 42, Issue 2, 86-91

Broderick, P. C. (2013). Learning to BREATHE: A mindfulness curriculum for adolescents. Oakland, CA: New Harbinger.

Calero, J. (2007). Desigualdades socio-económicas en el sistema educativo español. Madrid, Centro de Investigación y Documentación Educativa- Ministerio de Educación.

Cea. J.I., Caso, R., Reyes del Paso, GA., González-Pinto, A., Brazal, J., Martinez, B. (2005). Blood pressure is reduced after a breathing intervention in mild hypertensive patients. Society for Psychophysiological Research 45th Annual Meeting: 2005 September 21-24: Lisboa, Portugal

Cea, J.I. (2005). Abordaje psicofisiológico de la hipertensión arterial esencial. [Tesis Doctoral]. Universidad País Vasco.

Cea, J.I., González-Pinto, A., Cabo, OM. (2010). Respiración controlada para reducir el estrés. Estudio preliminar de su eficacia sobre el cortisol. Rev ROL Enf. 2010; 33:36874.

Conde, M., Menéndez, F.J., Santéd, M.A., Estrada, JD. (enero 1999). Incidencia de la frecuencia respiratoria en la conductancia eléctrica de la piel. Revista Electrónica de Motivación y Emoción. Disponible en: https://cutt.ly/Pt1RleI

Damasio, A. (2006). El Error de Descartes. Madrid, España: Crítica.

Davidson, Richard J. (2012). Contemplative Practices and Mental Training: Prospects for American Education. Child development perspectives, Volume 6, Number 2, 146153. 
Davidson, R. \& McEwen, B. (2012). Social influences on neuroplasticity: stress and interventions to promote well-being. Nat Neurosci, volume 15 | number 5 | MAY 2012.

Davidson, R. (2012). El perfil emocional de tu cerebro. Madrid, España: Destino.

Davis, M. (1980). A multidimensional aproach to individual diferences in empathy. JSAS Catalog os Selected Documents in Psycology, 10: 1-19

De Bono, E. (1989). El Pensamiento Lateral. Manual de Creatividad. Buenos Aires, Argentina: Paidós.

De Bono, E. (1992). El pensamiento práctico. Barcelona: Paidós.

Ekman, P., Keltner, D. (2014). Darwin's Claim of Universals in Facial Expressions Not Challenged. Huffington Post.

Ekman, P., Cordaro, D. (2011). What is meant by calling emotions basic. Emotion Review, 3(4), 364-370.

Franco, Mañas y Justo. (2009). Reducción de los niveles de estrés, ansiedad y depresión en docentes de educación especial a través de un programa de mindfulness. Universidad de Almería. Revista Educación Inclusiva, Vol.2 n³.

Gardner, M. (1985). Inspiración ¡Ajá!. Barcelona, España: Labor.

Hanson, Rachel. (2016). Yoga for Depression and Anxiety: A systematic review. Recuperado de Sophia, the St. Catherine University repository website: https://cutt.ly/pt1REol

Foray, D. y Lundvall, (1996). Employment and Growth in the Knowledge-Based Economy. Paris, France: B.A.

Golberg, E. (2002). El cerebro Ejecutivo. Madrid, España: Crítica.

Horkheimer, M. y Adorno, T. (2007). Dialéctica de la Ilustración. Madrid, España: Akal.

James, W. (1994). Principios de psicología. USA: Fondo de Cultura Económica.

Jensen, E. (2004). Cerebro y Aprendizaje: competencias e implicaciones educativas. Madrid, España: Narcea.

López González, L. (2016). Competencias de Relajación, Meditación y Mindfulness en la Escuela. Una propuesta de concreción. Universidad de Barcelona: ICE (prensa)

Maturana, A., Vargas, A. (2015). El estrés escolar. Revista Médica Clínica Las Condes, Volume 26, Issue 1, January.FebruaryPages 34-41.

Martínez-Otero Pérez, V. (2007). Estrés y ansiedad en los Docentes. Pulso. 20. 9-21.

Ma X, Yue Z-Q, Gong Z-Q, Zhang H, Duan N-Y, Shi Y-T, Wei G-X and Li Y-F. (2017). The Effect of Diaphragmatic Breathing on Attention, Negative Affect and Stress in 
Healthy Adults. Front. Psychol. 8:874. doi:10.3389/fpsyg.2017.00874. Recuperado de University repository website: https://cutt.ly/Rt1RIRs

Merleau-Ponty, M. (1985). Fenomenología de la Percepción. Barcelona, España: Planeta de Agostini.

Nietzsche, F. (1997). El crepúsculo de los ídolos. Madrid, España: Alianza.

Ortega y Gasset, José. (2012). El tema de nuestro tiempo. Obras completas. Vol. III. Madrid, España: Taurus.

Pascual-Leone, A., Sadato, J., Grafman, Ibañez, V. Deiber, NP., Dold, G. (1996). Activation of the primary visual cortex by Braille reading in blind subjects. Nature 380 (6574), 526-528.

Pascual-Leone, F. (1993). Plasticity of the sensorimotor cortex representation of the reading finger in Braille readers, Brain 116 (1).

Posner, M., Petersen, S. (1990). The attention system of the human brain. Annual Reviews of Neuroscience. 35, 73-89. https://dx.doi.org/10.1146 / annurev-neuro-062111-150525

Pert, C. (1999). The molecules of Emotion. New York: Touchtone Editions.

Ramachandran, V. S, (2012). Lo que el cerebro nos dice. Barcelona, España: Paidos.

Ramachndran, V. S. (2008). Laberintos del Cerebro. Barcelona, España: La Liebre de Marzo.

Ravinder, Braun, Barnes, Harden (2015). Self-Regulation of Breathing as a Primary Treatment for Anxiety. Revista Applied Psychophysiology and Biofeedback. 40 (2): 107-115

Ruiz Lázaro, PJ (2016). Mindfulness en niños y adolescentes. En: AEPap (ed.). Curso de Actualización Pediatría 2016. Madrid: Lúa Ediciones 3.0; pág. 487-501.

Sacks, O. (2006). Un antropólogo en Marte. Barcelona, España: Anagrama.

Satyananda, S (1966). Asana, Pranayama, Mudra, Bhanda. Munger, Bihar, India: Yoga Publications Trust. 